\title{
Spin Structure of the Proton and Transverse Momentum Dependent distributions
}

\section{A. Prokudin*}

Jefferson Lab, 12000 Jefferson Avenue, Newport News, VA 23606

E-mail: prokudinejlab.org

Transverse Momentum Dependent Distributions (TMDs) describe the spin structure of the proton. At leading twist spin structure of spin-1/2 hadron can be described by 8 TMDs. TMDs reveal three-dimensional distribution of partons inside polarised nucleon. Experimentally these functions can be studied in polarised experiments using Spin Asymmetries in particular Single Spin Asymmetries (SSAs). We discuss transversity that measures distribution of transversely polarised quarks in a transversely polarised nucleon and Sivers distribution function that describes distribution of unpolarised quarks in a transversely polarised nucleon.

35th International Conference of High Energy Physics - ICHEP2010,

July 22-28, 2010

Paris France

* Speaker. 
Transverse Momentum Dependent distribution functions (TMDs) depend on two independent variables: fraction of hadron momentum carried by parton, $x$, and intrinsic transverse momentum of the parton, $\mathbf{p}_{T}$.

The quark-quark distribution correlation function is defined as $[1,2]$

$$
\Phi_{i j}\left(x, \mathbf{p}_{T}\right)=\left.\int \frac{d \xi^{-} d^{2} \xi_{T}}{(2 \pi)^{3}} e^{i p \cdot \xi}\left\langle P, S_{P}\left|\bar{\psi}_{j}(0) \mathscr{U}_{(0,+\infty)}^{n_{-}} \mathscr{U}_{(+\infty, \xi)}^{n_{-}} \psi_{i}(\xi)\right| P, S_{P}\right\rangle\right|_{\xi^{+}=0}
$$

with $p^{+}=x P^{+}, P^{+}=\left(P^{0}+P^{3}\right) / \sqrt{2}$ is the big component of proton's momentum. $\mathscr{U}$ is the gauge link (Wilson line) that assures color gauge invariance of the correlator $\Phi_{i j}$.

Corresponding distribution functions can be obtained by projecting the correlator onto the full basis of $\gamma$ matrices $\Phi^{[\Gamma]}=\frac{1}{2} \operatorname{Tr}[\Phi \Gamma]$. At leading twist (expansion in $P^{+}$) the spin structure of the proton can be described by 8 TMDs (see $[1,2]$ and references therein):

$$
\begin{aligned}
\Phi^{\left[\gamma^{+}\right]}\left(x, p_{T}^{2}\right) & =f_{1}\left(x, p_{T}^{2}\right)-\frac{\varepsilon_{T}^{\rho \sigma} p_{T \rho} S_{T \sigma}}{M} f_{1 T}^{\perp}\left(x, p_{T}^{2}\right), \\
\Phi^{\left[\gamma^{+} \gamma_{5}\right]}\left(x, p_{T}^{2}\right) & =S_{L} g_{1 L}\left(x, p_{T}^{2}\right)-\frac{p_{T} \cdot S_{T}}{M} g_{1 T}\left(x, p_{T}^{2}\right), \\
\Phi^{\left[i \sigma^{\alpha+} \gamma_{5}\right]}\left(x, p_{T}^{2}\right) & =S_{T}^{\alpha} h_{1}\left(x, p_{T}^{2}\right)+S_{L} \frac{p_{T}^{\alpha}}{M} h_{1 L}^{\perp}\left(x, p_{T}^{2}\right) \\
& -\frac{p_{T}^{\alpha} p_{T}^{\rho}-\frac{1}{2} p_{T}^{2} g_{T}^{\alpha \rho}}{M^{2}} S_{T \rho} h_{1 T}^{\perp}\left(x, p_{T}^{2}\right)-\frac{\varepsilon_{T}^{\alpha \rho} p_{T \rho}}{M} h_{1}^{\perp}\left(x, p_{T}^{2}\right)
\end{aligned}
$$

here the projections are done with respect to the quark's polarisation, $\Phi^{\left[\gamma^{+}\right]}$is distribution of unpolarised quarks, $\Phi^{\left[\gamma^{+} \gamma_{5}\right]}$ is distribution of longitudinally polarised quarks and $\Phi^{\left[i \sigma^{\alpha+} \gamma_{5}\right]}\left(x, p_{T}^{2}\right)$ is the distribution of transversely polarised quarks.

A powerful tool to study the spin structure is measurement of spin asymmetries in the SIDIS process $\ell P\left(S_{P}\right) \rightarrow \ell^{\prime} h X$. A number of structures can be measured in DIS process $\ell P\left(S_{P}\right) \rightarrow \ell^{\prime} X$ or in SIDIS with two detected hardons $\ell P\left(S_{P}\right) \rightarrow \ell^{\prime} h_{1} h_{2} X$.

In case of SIDIS process $\ell P\left(S_{P}\right) \rightarrow \ell^{\prime} h X$ two scales exist: virtuality of the photon $Q^{2}$ and transverse momentum of the produced hadron $P_{h T}$ with respect to the lepton scattering plane. If the produced hadron has low transverse momentum $Q^{2}>>P_{h T}^{2} \geq \Lambda_{Q C D}^{2}$ then Transverse Momentum Dependent factorisation theorem [3] is valid. The cross section is written as convolution of TMDs $d \sigma^{\ell P\left(S_{P}\right) \rightarrow \ell^{\prime} h X} \propto \sum_{q} e_{q}^{2} f_{q} \otimes d \sigma^{\ell q\left(s_{q}\right) \rightarrow \ell^{\prime} q^{\prime}\left(s_{q^{\prime}}\right)} \otimes D_{q / h}$, where $\otimes$ stands for convolution of the transverse momenta. Experimentally TMDs can be measured using SSA on transversely polarised target defined as $A_{U T} \propto \frac{\sigma^{\uparrow}-\sigma^{\downarrow}}{\sigma^{\uparrow}+\sigma^{\downarrow}}$. Let us describe some of TMDs.

\section{Transversity}

Transversity distribution $h_{1}\left(x, p_{T}^{2}\right)$ [4] describes distribution of transversely polarised quarks inside a transversely polarised nucleon. Tensor charge measures net transverse polarisation of quarks

$$
\delta_{T} q=\int_{0}^{1} d x\left(h_{1 q}(x)-h_{1 \bar{q}}(x)\right)
$$

Transversity is a chiral-odd quantity and thus cannot be measured in DIS. In SIDIS transversity can be measured together with chiral-odd fragmentation function $H_{1 q}^{\perp}\left(z, k_{T}^{2}\right)$ the so-called Collins fragmentation function [5], the asymmetry is $A_{U T}^{\sin \left(\Phi_{h}+\Phi_{S}\right)} \propto \sum_{q} e_{q}^{2} h_{1 q} \otimes H_{1 q}^{\perp}$. 

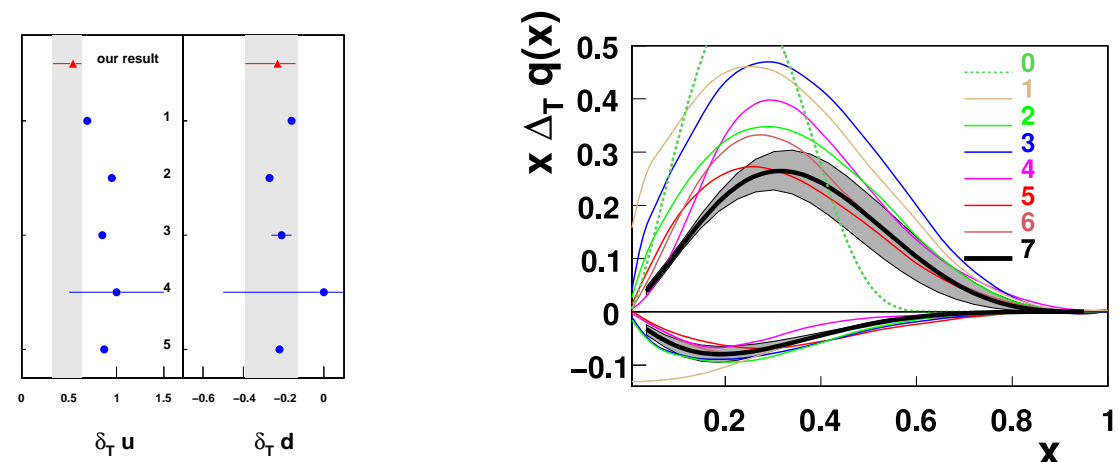

Figure 1: Left panel: extraction of tensor charge [10] compared to models [11]. Right panel: transversity for $u$ and $d$ quarks [10] compared to models [11].

The "golden" channel to measure transversity is double spin asymmetry in future Drell-Yan experiment with polarised proton and anti-proton beams, product of transversities can be measured [6] $A_{N N} \propto \sum_{q} e_{q}^{2} h_{1 q / P} h_{1 \bar{q} / \bar{P}}$.

Existing experimental data from HERMES, COMPASS and BELLE collaborations [7, 8, 9] allow extraction of transversity [10]. In Fig. 1 we plot tensor charge and transversity distributions compared to models. As can be seen from Fig. 1 the extraction and models agree qualitatively on the $u$ and $d$ quark transversities, but the experimental precision is still not good enough to discriminate among models.

\section{Sivers distribution function}

$f_{1 T}^{\perp}\left(x, p_{T}^{2}\right)$ is the so-called Sivers function [12], it describes correlation between orbital angular motion of quarks and the spin of the proton $\varepsilon_{T}^{\rho \sigma} p_{T \rho} S_{T \sigma}$. This function exists due to the presence of Final State Interactions of the struck quark and the remnant of the nucleon after the interaction, the corresponding asymmetry is $A_{U T}^{\sin \left(\Phi_{h}-\Phi_{S}\right)} \propto \sum_{q} e_{q}^{2} f_{1 T}^{\perp} \otimes D_{1 q}$.

This function obeys a modified universality, it changes sign from SIDIS to Drell-Yan [13]

$$
f_{1 T}^{\perp}\left(x, p_{T}^{2}\right)^{S I D I S}=-f_{1 T}^{\perp}\left(x, p_{T}^{2}\right)^{D Y},
$$

the prediction of change of sign based on color gauge symmetry and parity and time reversal invariance $\mathscr{P}, \mathscr{T}$ of strong interactions. Experimental test of this relation is very important for our understanding of QCD.

The first moment of Sivers function $f_{1 T}^{\perp(1)}(x)=\int d^{2} k_{\perp} \frac{k_{\perp}^{2}}{2 M^{2}} f_{1 T}^{\perp}\left(x, p_{T}^{2}\right)$ is related [14] to twist three Qiu-Sterman matrix element $T_{F}(x, x)$ [15]

$$
T_{F}(x, x)=f_{1 T}^{\perp(1)}(x) .
$$

Twist three matrix elements are ingredients of collinear QCD to describe spin asymmetries and the formalism is valid at high $P_{h T}>>\Lambda_{Q C D}$. The Sivers function describes the asymmetry at low values of $P_{h T} \geq \Lambda_{Q C D}$.

In Fig. 2 we plot Sivers function extracted from the experimental data [17, 8] a three dimensional parton distribution at $x=0.01$, as can be seen from Fig. 2 the distribution of partons in a transversely polarised hadron has dipole deformation. 

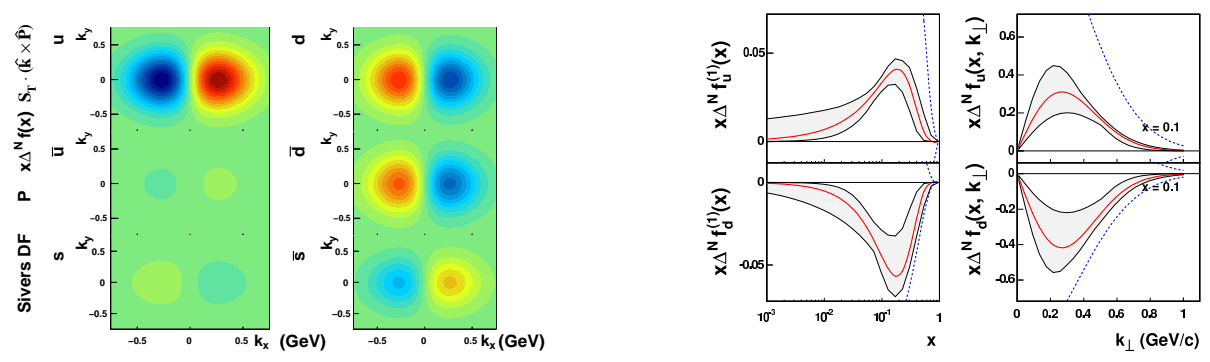

Figure 2: Left panel: three dimensional parton distribution. Right panel: extraction of Sivers function [16].

\section{Conclusions}

Transverse Momentum Dependent distributions describe spin structure of the proton. Experimental data from HERMES, COMPASS and BELLE collaborations allow extraction of transversity and Collins fragmentation functions. Sivers functions for $u$ and $d$ quarks were extracted from HERMES and COMPASS data. Future experimental data from JLab will widen explored $x$ region and refine precision of extraction of TMDs. $Q^{2}$ range at Electron Ion Collider will allow to check evolution of asymmetries, wide $P_{h T}$ range will allow to measure asymmetries in both regions where TMD and collinear QCD factorizations are valid.

\section{References}

[1] P. J. Mulders and R. D. Tangerman, Nucl. Phys. B461, 197 (1996).

[2] A. Bacchetta et al., JHEP 02, 093 (2007), hep-ph/0611265.

[3] X.-d. Ji, J.-p. Ma, and F. Yuan, Phys. Rev. D71, 034005 (2005), hep-ph/0404183.

[4] J. P. Ralston and D. E. Soper, Nucl. Phys. B152, 109 (1979).

[5] J. C. Collins, Nucl. Phys. B396, 161 (1993).

[6] PAX, V. Barone et al., (2005), hep-ex/0505054.

[7] HERMES, M. Diefenthaler, (2007), arXiv:0706.2242 [hep-ex].

[8] COMPASS, M. Alekseev et al., (2008), arXiv:0802.2160 [hep-ex].

[9] Belle, R. Seidl et al., Phys. Rev. D78, 032011 (2008), 0805.2975.

[10] M. Anselmino et al., AIP Conf. Proc. 1149, 465 (2009).

[11] http://indico.cern.ch/getFile.py/access?contribId=1059\&sessionId=46\&resId=0\&materialId=slides\&confId=73513 .

[12] D. W. Sivers, Phys. Rev. D41, 83 (1990).

[13] J. C. Collins, Phys. Lett. B536, 43 (2002), hep-ph/0204004.

[14] D. Boer, P. J. Mulders, and F. Pijlman, Nucl. Phys. B667, 201 (2003), hep-ph/0303034.

[15] J.-w. Qiu and G. Sterman, Phys. Rev. Lett. 67, 2264 (1991).

[16] M. Anselmino et al., Eur. Phys. J. A39, 89 (2009), 0805.2677.

[17] HERMES, A. Airapetian et al., Phys. Rev. Lett. 94, 012002 (2005), hep-ex/0408013. 